\title{
Modelo de Previsão de Vazão do Rio Madeira Aplicado ao Planejamento de Operação da Usina Hidrelétrica de Santo Antônio - Rondônia
}

\author{
Daylton Guedes e Osmar Pinto Junior, Instituto Nacional de Pesquisas Espaciais - INPE
}

Copyright 2016, SBGf - Sociedade Brasileira de Geofísica

\section{Resumo}

Um modelo de previsão de vazão desenvolvido por um projeto de Pesquisa e Desenvolvimento (P\&D) realizado pela empresa Santo Antônio Energia e pelo Grupo de Eletricidade Atmosférica (ELAT) do Instituto Nacional de Pesquisas Espaciais - INPE é apresentado. Usinas hidrelétricas de fio d'água dependem exclusivamente da vazão diária do rio, fato que torna este item um ativo de enorme importância na operação. A aplicação deste modelo se explica pela necessidade em atender exclusivas e complexas especificidades onde os parâmetros envolvidos são únicos. O objetivo é ter previsão de médio prazo, até três meses, com menores erros possíveis.

\section{Introdução}

O Brasil possui uma vasta área geográfica que comporta diversas bacias hidrográficas. O perfil do parque gerador de energia hidrelétrica tem sido modificado desde a última década com a incorporação das usinas que não possuem grandes reservatórios de água e que geram energia basicamente com a vazão dos rios. É o caso do rio Madeira, na região norte do país e da UHE Santo Antônio em Porto Velho - RO, considerando apenas as condições específicas na geração de eletricidade. $O$ modelo de previsão de vazão aproveita a série temporal de dados levantada em uma única estação por mais de quarenta anos. Para atender a estas necessidades, um código foi desenvolvido para determinar, estatisticamente, a vazão futura na calha do rio. A caracterização das propriedades de uma série temporal pode ser obtida considerando a variação dos seus parâmetros nos modelos matemáticos. Tendo obtido o padrão para uma determinada série temporal, para uma quantidade de dados observados, tal modelo pode então ser visto como definidor do processo preditivo (Wilks, 2011). Outrossim, a previsão de vazão de um rio feita a partir de séries temporais trata o problema como "uma caixa preta", sem fazer qualquer esforço no sentido de verificar quais fatores físicos afetam seu comportamento (Makridakes et al, 1983). É possível esperar que o histórico das medições de vazão possa conter informações importantes que definem o comportamento da calha do rio durante todas as estações do ano, apesar de que, precipitação na bacia hidrográfica, influências exteriores tais como degelo da cordilheira dos Andes e oscilações das temperaturas superficiais dos oceanos Pacífico e Atlântico e seus impactos sobre a circulação geral da atmosfera e, em decorrência, na precipitação na região de interesse, entre outras, ainda sejam objeto de estudos e têm sido discutidas. Por causa desta dinâmica, não se conhece em detalhes a importância relativa destes processos, bem como as equações que governam suas relações para a maioria das bacias hidrográficas brasileiras.

\section{Metodologia/ Problema Investigado}

Uma série temporal com cerca de quarenta anos de dados, com início em janeiro de 1975, foi utilizada. A série abrange medições diárias ininterruptas (leituras horárias), transformadas em vazões médias mensais como mostra a Figura 1, totalizando 492 médias mensais até 2015.

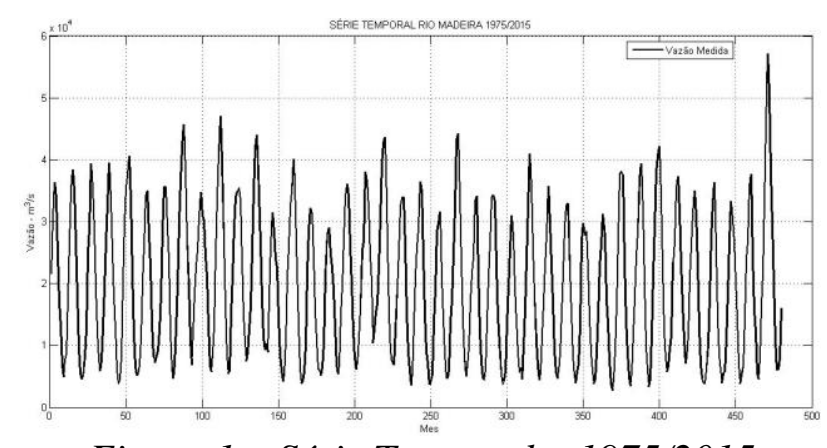

Figura 1 - Série Temporal - 1975/2015

Outra maneira de analisar os dados é comparar a variação das médias mensais durante o ano, como mostra a Figura 2, quando é possível identificar o padrão de comportamento da série.

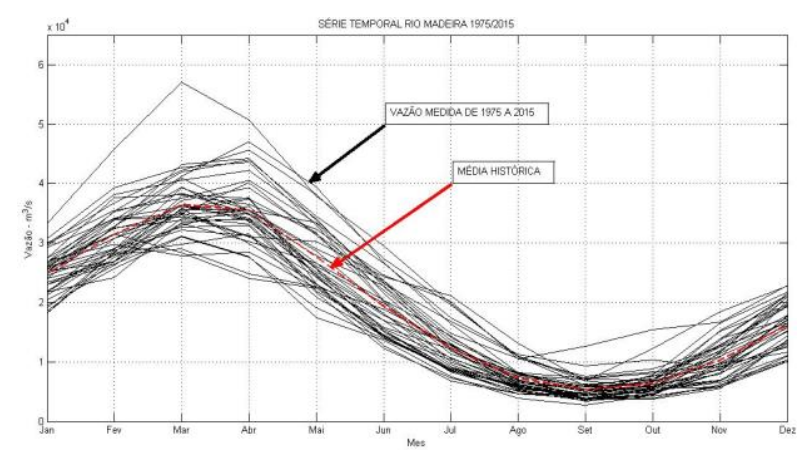

Figura 2 - Série Temporal - Variação mensal

Os modelos estatísticos podem ser probabilísticos/ estocásticos ou determinísticos/regressivos. As séries hidrológicas são, na maioria das vezes, séries estacionárias com leve tendência negativa, tem variações sazonais e não possuem periodicidades relevantes. Quando a análise de séries temporais mostra modelos auto regressivos, estes são considerados estocásticos e são amplamente utilizados nos estudos de modelagem de previsão de vazão (Wheellright et al., 1983). A série hidrológica das Figuras 1 e 2 mostra o crescimento da vazão nos meses de janeiro a abril e o decrescimento 
nos meses seguintes, com a menor vazão entre setembro e outubro. A série apresentou no verão do ano 2014 seu valor mais alto. Este comportamento reproduz o regime de chuvas na região norte do Brasil. O modelo estima vazões por trimestre. É de interesse prático que a previsão para um determinado trimestre seja feita no final do trimestre imediatamente anterior, quando os dados mensurados dos meses deste trimestre já devem estar disponíveis para uso. Esta estratégia permite ao planejamento da Usina tempo suficiente para programar a operação e manutenção contratada. Optou-se por calcular as constantes sazonais nos dois casos, determinístico e estocástico. Segundo Morettin e Toloi (2011), os métodos de regressão são ótimos para séries que representam sazonalidade determinística, ou seja, que possam ser previstas a partir de meses anteriores. $\mathrm{E}$ segundo MaKridakis et al, (1983), os modelos de multiregressão são apropriados para sistemas probabilísticos, leia-se, estocásticos. As constantes sazonais mensais, calculadas pelos dois métodos, são somadas separadamente às estimações de vazão. Acontece, entretanto, que, se duas séries temporais estão relacionadas entre si, seria incorreto considerar que o valor de uma das variáveis de uma das séries dependa de variações de uma variável da outra série. Tendo esta premissa em consideração, foi feito o cálculo da correlação entre as séries obtidas da série original para o melhor ajuste da previsão. Com a mesma finalidade (melhor ajuste), um tratamento semelhante foi dispensado à questão da variação das médias mensais, nas quais foram feitas, além das médias móveis centradas, em intervalo de um ano, as médias móveis deslocadas para o décimo terceiro mês a cada doze meses.

\section{Resultados}

De modo a verificar a possibilidade de que o contínuo degelo nos Andes verificado nas últimas décadas possa influenciar a vazão do rio Madeira no curto prazo, foi feito um estudo da literatura referente aos impactos projetados devido às mudanças climáticas sobre o ciclo hidrológico na Bolívia. As projeções ainda são em número reduzido e mostram variações em função dos modelos climáticos utilizados (Fry et al., 2010, Chevallier et al., 2011; Buytaert and De Bièvre, 2012; Lavado et al., 2012). Independentemente das incertezas, a diminuição significativa das geleiras dos Andes pode, em longo prazo, trazer uma redução na vazão dos rios andinos, entre eles o rio Madeira. O efeito, contudo, pode ser desconsiderado no contexto deste projeto, que visa à previsão de curto prazo (alguns meses) da vazão do rio Madeira. Quanto à previsão, o modelo desenvolvido foi testado para três casos distintos:

i) Ano de 2013, considerado um ano típico, cujos valores médios da vazão mensal não se distanciaram da média histórica da série; Ano de 2014, ano atípico, que teve nos meses de janeiro fevereiro e março as maiores médias desde 1975. Os valores das vazões de janeiro, fevereiro e março de 2014 são muito superiores às médias históricas da série. iii)

Ano 1998, que teve nos meses de setembro e outubro uma das menores médias desde 1975.

Com base na comparação dos anos 1998, 2013 e 2014, com seus valores já conhecidos, o modelo procurou testar a sua eficiência, ou seja, mostrar-se capaz de prever tais variações. Os cálculos foram feitos, primeiro negligenciando a sazonalidade, segundo, incluindo os efeitos da sazonalidade. No primeiro caso as curvas nem de longe reproduzem o comportamento comum das curvas anuais como mostradas na Figura 2. Já no segundo caso, usando os métodos determinístico e estocástico, os resultados coincidem, ou seja, o modelo descreve as curvas de forma idêntica, ainda que por métodos diferentes. Em todos os casos os cálculos mostram as previsões para os meses de janeiro a dezembro, feitas no final do trimestre imediatamente anterior ao primeiro mês do trimestre em estudo. A Figura 2 mostra a previsão de um ano 2013.

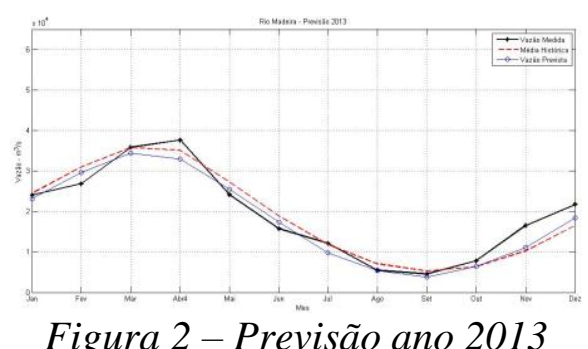

A Figura 3 mostra a previsão do ano 2014 e a máxima no mês de março, e a Figura 4 mostra a previsão do ano 1998 e sua mínima no mês de setembro.

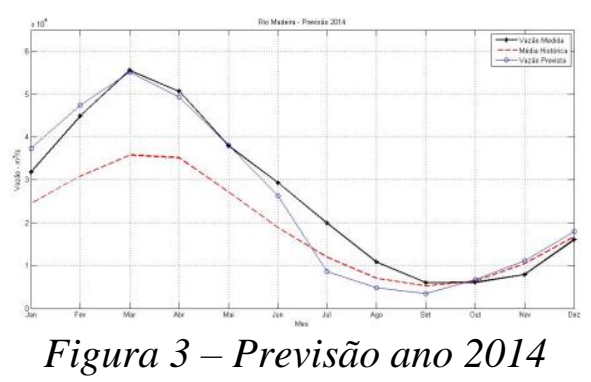

As medidas de precisão adotadas são semelhantes às utilizadas em previsões de séries temporais. Durante o desenvolvimento do modelo são reservados doze períodos, constituindo uma série para teste. Aqui, os anos de 1998, 2013 e 2014, respectivamente. A previsão é feita sobre os períodos da série teste. 


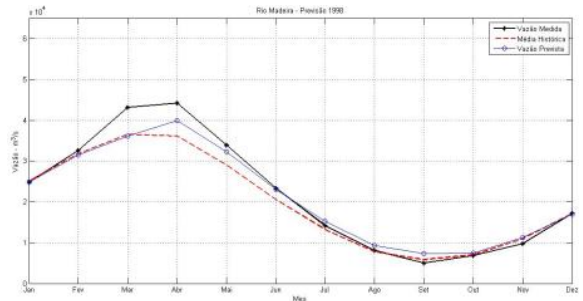

Figura 4 - Previsão ano 1998

A comparação entre os valores previstos e os dados reservados para teste determina a qualidade da previsão. São calculados dois erros: a média do valor absoluto do erro percentual - MAPE - que traduz o quanto as previsões se desviam percentualmente, em média, dos valores observados e a média dos valores absolutos dos desvios ao quadrado - EQM - que traduz o quanto as previsões de vazões se desviam em termos absolutos em relação aos valores observados. O exercício da previsão tem como objetivo estatístico aperfeiçoar os resultados sempre procurando um modelo que minimize o EQM. A tendência, entretanto, é que o EQM seja maior para rios com maiores vazões e menor para aqueles com menores vazões. De forma absoluta, quanto menor seja o valor do EQM, melhores são as previsões.

O MAPE é definido por:

$$
\text { MAPE }=\frac{1}{H} \sum_{i=1}^{H} \frac{\left|Y_{i}-\hat{Y}_{i}\right|}{Y_{i}} x 100
$$

O EQM é definido por:

$$
E Q M=\frac{1}{H} \sum_{i=1}^{H}\left(Y_{i}-\hat{Y}_{i}\right)^{2}
$$

onde $H$ é número total de períodos estimados (i) usados na previsão, $Y_{i}$ é a vazão média mensal observada (reservada para teste) no período i e $\hat{Y}_{i}$ é a vazão média mensal estimada no período i.

A Tabela 1 mostra os erros MAPE e EQM para os anos em estudo.

\section{Discussão e Conclusões}

O Sistema Interligado Nacional- SIN mantem vários modelos de previsão de vazão para as diversas bacias hidrográficas do país. Em alguns casos, os erros encontrados, considerando os modelos que funcionaram entre 1997 e 2004, ficaram entre $12 \%$ e $68 \%$ e no período $2000 / 2001$, entre $10 \%$ e $52 \%$ (Costa et al, 2007). Não se trata aqui de comparar modelos, pois esses modelos foram concebidos sobre bases e aplicações diferentes. Os modelos usados pelo Operador Nacional do Sistema Elétrico - ONS são mais amplos quanto a aplicação e tem utilizadas as previsões de precipitação geradas e disponibilizadas pelo CPTEC/INPE (modelo ETA). Já o modelo aqui apresentado foi desenvolvido para uma aplicação muito específica. Tendo isto em mente, os erros mínimos encontrados para os anos 1998, 2013 e 2014 foram $0,50 \%, 2,12 \%$ e $0,45 \%$ respectivamente.
Tabela 1 - Erros de Previsão para os anos1998,2013 e

\begin{tabular}{|c|c|c|c|c|c|c|}
\hline \multicolumn{7}{|c|}{ Erros MAPE E EQM PARA AS PREVISÕES DOS ANOS 1998, 2013 E 2014} \\
\hline Ano & \multicolumn{2}{|c|}{1998} & \multicolumn{2}{|c|}{2013} & \multicolumn{2}{|c|}{2014} \\
\hline Mês & $\begin{array}{l}\text { MAPE } \\
(\%)\end{array}$ & EQM & $\begin{array}{c}\text { MAPE } \\
(\%)\end{array}$ & EQM & $\begin{array}{l}\text { MAPE } \\
(\%)\end{array}$ & EQM \\
\hline Janeiro & 0,50 & 15.650 & 3,75 & 813.694 & 17,38 & 30.604 .351 \\
\hline Fevereiro & 3,24 & 1.117 .185 & 10,40 & 7.797 .330 & 5,59 & 6.314 .415 \\
\hline Março & 16,17 & 48.740 .783 & 3,94 & 1.990 .836 & 0,86 & 228.751 \\
\hline Abril & 9,70 & 18.374 .339 & 12,46 & 22.038 .705 & 2,64 & 1.791 .930 \\
\hline Maio & 4,59 & 2.420 .575 & 5,53 & 1.785 .884 & 0,45 & 28.964 \\
\hline Junho & 0,92 & 46.134 & 10,60 & 2.782 .757 & 10,37 & 9.239 .289 \\
\hline Julho & 7,86 & 1.240 .327 & 19,04 & 5.350 .986 & 56,46 & 126.579 .600 \\
\hline Agosto & 15,43 & 1.557 .528 & 2,12 & 13.588 & 54,80 & 35.403 .333 \\
\hline Setembro & 45,72 & 5.224.515 & 18,36 & 707.566 & 41,88 & 6.407 .834 \\
\hline Outubro & 9,62 & 429.614 & 17,08 & 1.775 .636 & 9,76 & 354.346 \\
\hline Novembro & 15,92 & 2.410 .722 & 32,70 & 29.286.821 & 40,82 & 10.457 .527 \\
\hline Dezembro & 0.84 & 20.753 & 15,15 & 10.861 .111 & 11,81 & 3.578 .415 \\
\hline
\end{tabular}
2014

Já os erros máximos encontrados para os mesmos anos em estudo foram $45,72 \%, 32,70 \%$ e $56,46 \%$. Há uma tendência de regionalização da previsão climática no âmbito do SIN que impacta com certeza, a previsão de vazão. Para a aplicação na hidrologia há pleno potencial de utilização dessas estimativas climáticas regionais de chuvas na área de previsão de vazões e no aprimoramento do conhecimento sobre os diferentes fenômenos naturais que constituem o ciclo hidrológico. Entretanto, o modelo apresentado, por utilizar uma base extensa de médias mensais, cerca de 492 meses, não trata separadamente a contribuição da precipitação climática, influências externas como variação de temperatura oceânicas ou movimento das massas de ar pelo continente. A contribuição do modelo nos cálculos de previsão de vazão é a aplicação de ferramentas estatísticas para fazer ajustes no mecanismo de cálculo considerando que essas contribuições estão contidas nos meses que compõem a série. É possível estender os cálculos para previsões mensais, de forma a minimizar o erro, já que quanto mais perto do ponto de observação, menor é o erro de estimativa, da mesma forma para previsões diárias, que neste caso implica em uma modificação nas rotinas de cálculos já estabelecidas.

\section{Referências}

Buytaert, W. and B. De Bièvre, Water for cities: The impact of climate change and demographic growth in 
the tropical Andes. Water Resources Research, 48(8), 2012, 13pp.

Chevallier, P., B. Pouyaud, W. Suarez, and T. Condom, Climate change threats to environment in the tropical Andes: glaciers and water resources. Regional Environmental Change, 11(S1), 179-187, 2011.

Fry, L., D. Watkins, J. Mihelcic, N. Reents, Sustainability of gravity fed water systems in Alto Beni, Bolivia: preparing for change, Proceedings of the World Environment and Water Resources Congress, Providence, Rhode Island, USD, May, 2010.

Lavado, C.W.S., J. Ronchail, D. Labat, J.C. Espinoza, and J.L. Guyot, Basin-scale analysis of rainfall and runoff in Peru (1969-2004): Pacific, Titicaca and Amazonas drainages. Hydrological Sciences JournalJournal Des Sciences Hydrologiques, 57(4), 625-642, 2012.

MAKRIDAKIS, S.; WHEELWRIGHT, S. C.; McGEE, V. E. Forecasting: Methods and Applications. 2a ed.. Nova York: John Wile \& Sons, 1978, 1983.

MORETTIN, P. A. e TOLOI, C. M. C. (2011). Análise de Séries Temporais. BLUCHER.

Wheellright, S. C., S. Makridakis, and V. E. McGee, Forecasting: Methods and Applications, John Wiley \& Sons, New York, 1983.

Luiz Guilherme Ferreira Guilhon, Vinicius Forain Rocha e Jonatas Costa Moreira - Comparação de métodos de previsão de vazões naturais afluentes a aproveitamentos hidroelétricos.

Daniel S. Wilks - Statistical methods in the atmospheric sciences $3 \mathrm{rd}$ ed. p.cm - (International geophysics series; v. 100) ISBM 978-0-12-385022 (alk. paper), 2011

Fernanda da Serra Costa, Maria Elvira Piñeiro Maceira, Jorge Machado Damázio - Modelos de Previsão Hidrológica Aplicados ao Planejamento da Operação do Sistema Elétrico Brasileiro - Universidade do Estado do Rio de Janeiro - UERJ 